\title{
Théologiques
}

Revue interdisciplinaire d'études religieuses

Théologiques

\section{Ultime fragilité et sublime espérance : l’expérience de la mort}

\section{Raymond Lemieux}

Volume 28, numéro 1, 2020

Pour une culture palliative ? Fragilités et vulnérabilité

URI : https://id.erudit.org/iderudit/1074677ar

DOI : https://doi.org/10.7202/1074677ar

Aller au sommaire du numéro

Éditeur(s)

Institut d'études religieuses de l’Université de Montréal

ISSN

1188-7109 (imprimé)

1492-1413 (numérique)

Découvrir la revue

Citer cet article

Lemieux, R. (2020). Ultime fragilité et sublime espérance : l'expérience de la mort. Théologiques, 28(1), 97-126. https://doi.org/10.7202/1074677ar

\section{Résumé de l'article}

L'espérance est cette vertu propre à l'humain qui lui permet de concevoir un monde différent et l'engage à travailler à son avènement. Que peut-il advenir, dès lors, dans ce passage ultime de sa vie où il est confronté à la mort ? Trois facettes de la question sont explorées : comment se présente désormais l'expérience de la mort ? Comment s'y greffe l'espérance, anthropologiquement et théologiquement ? Comment le fait de vivre appelle une ouverture à l'altérité qui est comme pare-feu à l'encontre des pulsions mortifères ? Le défi de la vie n'est-il pas dès lors d'espérer, malgré tout ? 


\title{
Ultime fragilité et sublime espérance : l'expérience de la mort
}

\author{
Raymond LEMIEUX" \\ Faculté de théologie et de sciences religieuses \\ Université Laval, Québec
}

Au moment d'entreprendre l'écriture de ce texte, le journal quotidien signale une découverte archéologique inusitée : dans le monde romain préchrétien, des rituels funéraires auraient été pratiqués pour des jeunes enfants, voire des foetus. La découverte bouleverse des idées reçues : dans cette civilisation, les enfants en bas de quatre ans n'avaient pas d'existence juridique. Que signifie, dès lors, l'évidence d'inhumations rituelles et de soins apportés à leurs restes, déposés dans des coffres imputrescibles au point de résister à deux millénaires d'enfouissement ? Au-delà la curiosité archéologique, c'est la conception même de l'humain chez nos lointains ancêtres qui est mise en question : ritualiser les restes d'un vivant, fussent-ils poussière, c'est lui donner consistance au-delà des limites de sa vie, c'est faire confiance à l'altérité, à ce qui n'a pas de mot pour se dire ni d'image pour s'imposer. C'est miser sur un réel impondérable, bref, c'est espérer.

En christianisme, la vertu de religion est une posture éthique - non pas une position acquise, mais bien une posture - qui consiste à miser ainsi sur l'Autre, sans relâche, à le ré-élire (Allard 2001), pour aller de commencement en commencement par des commencements qui n'ont jamais de fin (Grégoire de Nysse 2009, 109). Cette posture ne peut être réduite à une morale, fût-elle harmonieuse et désirée de tous, elle n'est pas une gestion des mœurs. Elle est mouvement, tension, dépassement vers un horizon qui s'éloigne à mesure qu'on pense s'en approcher. À la mesure de la vie, le salut ne réside pas dans un lieu : il s'élabore au rythme d'une marche. Sur

* Professeur émérite. A enseigné la sociologie de la religion, l'histoire du christianisme médiéval et moderne, de même que l'épistémologie des sciences de la religion. Premier titulaire de la Chaire Religion, spiritualité et santé de l'Université Laval.

(C) Revue Théologiques 2020. Tout droit réservé. 
les chemins de terre et les chemins de foi, dans la plaine comme à la montagne, il suppose de procéder pas à pas : un paso mas alla (Elizondo 2002), ultreïa ${ }^{1}$.

Sur la route montante, traînée, pendue aux bras de ses deux grandes sœurs qui la tiennent par la main, la petite fille espérance s'avance.

Entre ses deux grandes sœurs elle a l'air de se laisser traîner, comme une enfant qui n'aurait pas la force de marcher et qu'on traînerait sur cette route malgré elle.

Et en réalité c'est elle qui fait marcher les deux autres. Et qui les traîne, et qui fait marcher tout le monde, et qui le traîne. (Péguy 1992, 292)

La petite fille espérance, comme l'appelle Péguy, engage et entraîne chacun au cœur de son humanité. Le poète, ici, inscrit dans le langage une expérience qu'entérineront volontiers théologiens et anthropologues, voire tous ceux qui, d'une façon ou d'une autre, ont la passion de l'humain. L'humanité ainsi considérée n'est pas figée, ce n'est pas non plus une humanitéconcept, mais elle se livre dans le cœur palpitant d'une expérience, un espace-temps intime dans lequel rien n'est acquis. Dans le présent fugace de cette expérience se jouent le sens que chacun attribue à son passé de même que l'avenir sur lequel il mise. De là la beauté de ses visages : même balafrés par la vie ils restent florissants de promesses, fût-ce le temps d'un sourire. «Imprédictible beauté », selon le mot de Patrick Chamoiseau $(2018,107)$, qui ne peut être calculée ni programmée et se révèle dans la bienveillance d'un regard. Fulgurante beauté qui, enchaînée aux lourdeurs de l'histoire, y prend appui pour s'élancer vers l'altérité, et fait ainsi signe du dépassement de la mort dans le désir de vivre malgré tout, jusqu'à ériger ce signe en objet de contemplation. En effet, c'est souvent sur le point précis des traumatismes de la vie qu'elle se manifeste en splendeur, quand elle se donne par exemple dans le visage du sans-abri, du marginal, du fou, du laissé pour compte. Elle appartient ainsi à l'humanité en marche, cette humanité protéiforme dont l'une des dernières célébrations monumentales

1 Cri et chant joyeux médiéval encore en vogue sur les chemins de Compostelle. 
se trouve dans la sculpture de Timothy Schmalz ${ }^{2}$ installée récemment sur les terres vaticanes.

Foi et charité, les deux grandes sœurs de l'espérance, tiennent celleci par la main. Elles sont des tutrices solides, argumente encore Péguy, parce qu'elles voient et aiment "ce qui est », alors que leur petite compagne, elle, «voit ce qui sera». Elle anticipe l'à-venir, "pour ainsi dire le futur de l'éternité même ", insiste-t-il. Mieux que les savants pour qui les mots ont d'abord une fonction utilitaire, le poète ouvre ici un programme de vie, un véritable programme sémiotique qui engage la dynamique des signes par lesquels le monde devient présent à la conscience. Ce programme en est un de civilisation. Son dynamisme dépend des expériences concrètes de la vie et sa force propulsive est la contemplation.

Tentons à notre tour d'en saisir quelques éléments, dans un parcours en trois temps représentant autant d'étapes, structurelles et structurantes, qui jalonnent les élans spirituels de l'humain et déterminent leurs bases anthropologiques : mourir, espérer, vivre.

\section{Mourir}

«La mort ne se sent que par le discours, d'autant que c'est le mouvement d'un instant ». Cette sentence de Montaigne $(1962,55)$ résume bien la problématique qu'il nous faut élaborer : chacun ne conçoit sa propre mort que dans son occurrence unique, à venir. Quand cette occurrence est passée, il n'est plus là pour en parler. Il doit donc l'incorporer dans un discours anticipateur. Aussi ce discours concerne-t-il toujours la mort des autres, celle qu'on garde en mémoire, celle que la culture ambiante rappelle par des paroles, des monuments, des rituels... Tous ces lieux de mémoire, bien sûr, doivent être traités avec prudence car ils traduisent tout autant les intérêts de ceux qui les entretiennent aujourd'hui que la réalité perdue du passé, reconstituée au bénéfice du présent ${ }^{3}$.

L'acte de mourir fait ainsi événement : il est rupture / structure. Pour ceux qui restent, il est déchirure du tissu relationnel dans lequel ils étaient

2 «Angels Unaware », sculpture inaugurée le 30 septembre 2019. Pour quelques prises de vue en ligne : Timothy Schmalz Angels Unaware.

3 Pour s'en faire une idée actualisée, voir le roman pamphlet du philologue Claude Duneton (2004). 
jusque-là engagés, tissu qu'ils doivent dès lors s'appliquer à raccommoder par un travail de deuil. Pour ceux qui partent, ou qui perçoivent l'imminence du voyage - les moritori ${ }^{4}$ - il fait brèche dans la suite du monde. Il leur est impossible de trouver refuge dans du connu : ils ne peuvent rien savoir des territoires de l'altérité dans lesquels la mort les propulsera. Ils sont astreints à saluer l'instance plus grande qu'eux-mêmes qui préside au déroulement de leur histoire. Ils ne peuvent alors que conjecturer l'au-delà en s'appuyant sur le croyable disponible dans leur communauté et leur environnement culturel, ou plus largement sur le territoire de leurs habitus, leur milieu de vie, la catégorie sociale à laquelle ils s'identifient, voire le «sens commun», désormais objet médiatique émergeant d'une culture mondialisée.

Pour se donner le courage d'une traversée des limites dont il ne connaît pas l'issue, voire continuer de vivre, ne fut-ce que quelques minutes, celui qui va mourir ne peut qu'imaginer les territoires de l'altérité qui lui échappent. C'est pourquoi la plupart des humains, même quand leur vie a été pétrie d'horreurs - du moins à leur jugement -, choisiront à l'approche de la mort de continuer de vivre.

Survivre, en effet, implique une production de sens à double volet: d'une part, continuer de vivre (living on), d'autre part vivre ailleurs et autrement. Dans un cas comme dans l'autre, l'intensité émotionnelle accompagnant l'approche de la mort active le travail de représentation, chez le mourant comme chez ceux qui restent : il s'agit de concevoir l'à-venir, indéfini, en lui donnant un maximum de vraisemblance et, pour cela, le faire advenir au langage, selon le sens le plus élémentaire du mot inventer, invenire. Il faut faire venir, dans ce qui peut être dit et partagé avec d'autres humains, des éléments symboliques et imaginaires : mots, gestes, images, représentations - qui colmateront quelque peu les blessures du vécu et rendront attrayante la perspective d'une autre vie. Autrement dit, il s'agit d'inscrire l'expérience en train d'être vécue dans le registre de la convivialité, voire de la solidarité qu'appellent des liens sociaux qui, chez l'humain, à la différence des autres animaux - à moins que l'éthologie ne prouve un jour le contraire - ne sont pas génétiquement déterminés mais doivent être cultivés et soignés pour délivrer leur potentiel. Dans tous les cas, la survie est

4 Ave Caesar, morituri te salutant (Salut, César, ceux qui vont mourir te saluent). 
une concrétisation de l'espérance, un objet qui lui est donné, à la fois produit du désir du sujet et formaté par son environnement culturel.

De là l'importance du prendre soin ${ }^{5}$ dans les dispositifs d'accompagnement déployés autour de l'acte de mourir. Ce prendre soin dépasse bien évidemment les objectifs, certes toujours nécessaires, du curatif. Alors que celui-ci déploie technologies et protocoles visant à adoucir les aspérités de la route, à maîtriser les étapes du voyage, voire à déterminer le moment opportun de son issue, prendre soin exige un autre type de rigueur : prévenance, responsabilité, attention éducative, compassion, attention aux besoins des autres... (Tronto 2009). Ces exigences éthiques ne discréditent en rien la rigueur technique nécessaire aux protocoles curatifs, au contraire elle les incorpore en les inscrivant dans une visée plus large. Il y a complémentarité nécessaire entre attention à l'humain et rigueur technique, visée humaniste et responsabilité opérationnelle. La première donne sens à la seconde et la seconde atteste le sérieux de la première. Le bon Samaritain ne se contente pas d'avoir pitié du blessé de la route, il en prend soin concrètement, allant jusqu'à engager ses deniers pour lui assurer logement, nourriture, médicaments... Il ne choisit pas entre le caring et le curing mais inscrit dans la charité une rationalité qui, bien que toujours limitée, la rend tangible. Plutôt que tourner à vide, sa compassion prend alors tout son sens.

Mais il est une autre dimension du désir de survivre, elle aussi expression d'espérance aux assises anthropologiques, semble-t-il, universelles. Elle consiste à ouvrir la possibilité, pour le mourant, d'accéder à une autre vie après la mort, une vie qui, alors même qu'il affronte les limites actuelles de sa vie, en présente en quelque sorte le miroir inversé. Le concept de vie éternelle traduit particulièrement bien cet espace-temps sans limite, sans manque ni attente, où le désir devient pure béatitude et le bonheur définitif.

Les religions prennent généralement en charge ce rapport à l'Autre. Elles lui prêtent figure, instituent le Nom du Père à la place de l'infondé et organisent en système ce qui autrement resterait chaos. Elles ouvrent le temps clos de la réalité à un dynamisme toujours renouvelable puisque, par

5 On peut penser que l'augmentation du nombre des personnes âgée dans le monde occidental, augmentation liée au baby boom de l'après-guerre, a quelque peu pris de court cette exigence du prendre soin, faisant de ces populations des foyers de vulnérabilité sociale et sanitaire. 
définition, sans fin. L'indéfini du sens devient alors sensé : il prend figure opérationnelle capable de traduire le désir en mobilisations effectives. Ainsi mises en rapport avec la vie éternelle, les actions concrètes d'ici-bas peuvent trouver un plus-de-sens malgré les traumas de la rupture et l'arrière-goût des désenchantements. Il s'agit toujours de continuer de vivre, mais en entérinant la rupture, une fois traversées les limites qu'impose la mort. Donc non plus survivre dans la continuité du risque de mourir, mais expérimenter une autre vie, au-delà de la mort.

Sous une forme ou une autre, la sublimation de la mort, n'a rien de neuf et, sans doute, continue de produire partout un idéal du bien mourir, de façon à trouver une entrée adéquate dans l'autre vie. Dans les sociétés nobiliaires et guerrières du Moyen Âge, pour ne pas remonter plus loin, l'idéal d'une bonne mort se présentait sur le champ de bataille, le champ d'honneur dit-on encore aujourd'hui, en train de défendre une noble cause dans la boue, les larmes, le sang, les entrailles déchirées et exposées, c'est-àdire l'explicitation spectaculaire du chaos et du non-sens. La mort idéale se vivait alors dans la dramatique d'une existence où le sujet, fort de sa dignité et de la valeur de sa cause, mettait sa vie en jeu pour que sa vie ait du sens. Avec l'avènement de la bourgeoisie comme catégorie sociale hégémonique, dans la modernité occidentale, un autre modèle s'est petit à petit imposé (Ariès 1967 ; 1975 ; Thomas 1975) La «bonne mort » du bourgeois, celle pour laquelle on prie dans les foyers, se veut une mort très douce (Beauvoir 1964), quitte à en cacher le caractère inéluctable à celui ou celle qui la vit, sans esclandres, au milieu d'une communauté plus ou moins étendue de proches, assistée des secours sinon de la religion, du moins d'une conscience apaisée. On ne dénie pas le caractère dramatique de l'événement - de toute façon, comment pourrait-on l'oublier ? - mais on peut faciliter l'avènement de l'inconnu en supportant moralement le mourant. Les solidarités alors en jeu ne sont certes pas celles des frères d'armes sur le champ de bataille : elles relèvent d'un autre type de relations, renvoyant au destin commun, voire à l'héroïsme de la vie ordinaire ${ }^{6}$. L'espérance peut y être enchâssée ou non dans des discours explicitement religieux. Peu importe : devant l'inconnu qui vient, elle étale des pratiques d'accompagnement qui vont de la compassion jusqu'aux techniques de pointe dans la dispensation de soins curatifs.

6 Que mettront volontiers en valeur les eulogies dans les cérémonies de funérailles. 
Le monde contemporain aux frontières devenues poreuses - modernité avancée et ultramodernité - est largement héritier de la bourgeoisie occidentale. Aussi propose-t-il l'idéal d'une mort douce, sans souffrance, et continue-t-il de mettre au point une panoplie de moyens techniques pour y parvenir. Certes il ne néglige pas l'attention à l'humain et, quand cela est possible, valorise le fait d'être " accompagné des siens » : les notices nécrologiques dans les journaux témoignent régulièrement du fait que la personne décédée a été « accompagnée de ceux qu'elle a aimés ", sans oublier de souligner la compétence et les qualités du personnel soignant. Faisant intervenir des spécialistes du soutien émotionnel quand cela est utile, le monde contemporain cherche à atténuer les frontières entre le curatif et le caritatif parce que celles-ci, à l'évidence, sont blessantes. À la différence des contextes traditionnels, les solidarités dites «naturelles » (famille, proches) y sont de plus en plus aléatoires et les solidarités affinitaires (fondées sur les styles de vie, les goûts ou les orientations socioaffectives) sont fragiles et fluctuantes. Chacun ayant été habitué à concurrencer tous les autres pour faire sa place, chacun est destiné à mourir plus ou moins seul. Dans les meilleurs cas, un accompagnement spirituel lui est proposé, sous forme d'intervention professionnelle spécialisée dans l'accompagnement de fin de vie, de façon à ce que le care, institutionnalisé, cohabite aussi harmonieusement que possible avec la cure.

Bref, plus les sociétés semblent avancer vers l'ultramodernité, plus l'acte de mourir devient étranger à la vie. Que l'on soit riche ou misérable, la mort devient accidentelle : soit par un coup du sort sur la route, le mauvais fonctionnement d'une machine quelconque (au premier chef : le corps et ses organes), voire la perte de contrôle de soi... Elle est l'accident d'un ordre (une médecine, notamment) qui n'a pas encore trouvé le moyen de la vaincre, mais y travaille avec acharnement. Quand elle survient, c'est que quelque chose a échappé à la maîtrise rationnelle-technique du monde : cela est conjoncturel, bien sûr, et susceptible d'être corrigé.

Pour nos parents et grands-parents, croyants ou non, le salut était une notion essentiellement religieuse. Fleurons de la modernité, les révolutions bourgeoises du dix-huitième siècle en ont d'abord sécularisé la dimension politique, puis des habitus se sont imposés pour en séculariser les implications personnelles. On peut dire aujourd'hui que le salut de l'individu, de plus en plus, « consiste dans la libération de l'emprise maléfique 
de l'ignorance, de la superstition et de l'acceptation fataliste des oppressions naturelles ou sociales. La raison, la science et la technique [sont] les agents de cette libération, de ce salut séculier» (Fuchs 1998, 139). Une telle sécularisation du salut suppose un déplacement du sens de l'acte de mourir et implique la transformation des discours de survie, quelle que soit leur provenance.

L'inscription du mourir dans la logique procédurale peut aller loin. Elle peut proposer des enjeux performatifs qui sont non seulement ceux du noble guerrier rêvant de quitter ce monde l'épée à la main ou du kamikaze programmant le spectacle de sa sortie pour enfin être reconnu (Sutter 2016), elle peut aussi représenter, pour le bourgeois de classe moyenne, une dernière étape de sa croissance (Kübler-Ross 1975).

La question du salut y est donc fondamentale et multiforme. Sécularisé et désenchanté, le citoyen ultramoderne cherche frénétiquement à échapper aux fatalités que représentent la souffrance, la violence, les impasses de la vie collective et la solitude. Il rêve de mourir non seulement sans souffrance mais à un âge avancé et, surtout, sans dégradation psychique. Il compte sur l'efficacité de ses remises en forme pour atténuer les effets du vieillissement. Il vise à éviter toute forme de violence, tant celle, irrationnelle, des autres humains que celle, systémique, d'environnements rébarbatifs. Bref, la survie suppose un monde dans lequel tant la nature que la sensibilité de chacun seraient respectées et où les Léviathans seraient mâ̂trisés.

Traditionnellement, c'est le travail civilisateur des religions qui prenait en charge ce rapport à la survie. Mais quand l'imaginaire religieux est oublié, comme c'est le cas dans les sociétés sécularisées, d'autres discours tendent à prendre la relève, remplissant grosso modo les mêmes fonctions en utilisant des matériaux différents. La majorité de nos contemporains ne croient plus en Dieu mais ils disent volontiers de leur proche disparu : «Il a enfin trouvé la paix... il nous regarde et veille sur nous... ». Ils ne savent plus grand-chose des Trônes, Dominations et autres hiérarchies propres à l'organisation sociale du paradis, mais ils reconnaissent volontiers un ordre cosmique capable de s'imposer au monde et d'articuler passé, présent et avenir dans un même destin. Ils ont oublié les saints patrons de leurs ancêtres, mais ils échappent difficilement aux représentations laiques, séculières et marchandes d'un cosmos « capable d'assumer sa permanence face 
au chaos» (Berger 1971, 94). Ils lui demandent de garantir les orientations de leurvie.

Certes, la distinction peut paraître ténue entre les religiosités d'autrefois et les sécularités religieuses d'aujourd'hui (Lemieux 1999). Inventer une survie après la mort, c'est-à-dire la faire advenir au langage, c'est aussi se donner la possibilité de continuer de vivre, ici-bas et maintenant. Assumer la mort, figure archétype de toutes les limites de l'existence, continuer de vivre en lui faisant face, n'est-ce pas dès lors choisir « la vie la plus intense possible»(Derrida 2004, 8) ? Et qu'est-ce que la vie la plus intense possible sinon celle qui accepte la vérité de ses limites ? Contrairement à ce qu'on pourrait penser, c'est quand il est menacé, et non quand il est assuré, que le sens devient un enjeu de vie. C'est dans le trauma que la beauté devient admirable. C'est dans la crise que l'espérance prend corps. Cela se vérifie pour la rationalité capitaliste elle-même, quand l'anarchie sociale provenant de ses propensions destructrices la pousse, fût-ce timidement, à se remettre en question.

Dans son Introduction aux existentialismes, Emmanuel Mounier (1962) évoque une double contingence à propos de l'existence humaine. La première renvoie au mystère de chaque sujet qui tente de frayer son chemin dans les foisonnements du croyable : une "forêt de symboles", avait déjà dit Baudelaire (1991, 62). La seconde concerne le mystère en général, le sens commun que cultivent les mythes, anciens et modernes, et dont l'astuce, précisément, consiste à se présenter comme « naturel » alors qu'il est construction culturelle (Geertz 1986, 108-110). À cette double contingence se conjuguent des modes différenciés de gestion de l'espérance (et de son corollaire le désespoir) : l'un concerne les désirs relatifs qui mobilisent l'existence au quotidien et dont l'effondrement génère des crises parce qu'alors plus rien ne tient dans les montages du sens sur lesquels on comptait. L'autre concerne le rapport à l'altérité en tant que telle, l'instance de l'Autre. Non sans accent kierkegaardien, Mounier parlera de «désespoir fini » et de «désespoir infini ». Au «mal à l'âme » quelque peu égocentrique se surimpose ainsi un "gémissement de l'être vers l'être » qui intègre et sublime les malaises circonstanciels, c'est-à-dire les porte au-delà des limites de la culture. 
Un événement - rupture / structure - est aussi un avènement, une promesse. Devant la mort, le passé est fini et le présent inéluctable ; l'humain ne peut rien y changer. Lui reste l'à-venir, immédiat ou lointain, qui peut s'avérer promesse, investissement de son désir.

On sait comment l'imminence du mourir, désormais techniquement contrôlable dans les protocoles curatifs, suscite de nombreux débats. Jusqu'où, par exemple, est-il pertinent (licite, légal, moral, éthique) de prolonger la vie d'un patient ou de laisser l'usure de l'organisme faire son œuvre «naturellement» ? Le prolongement des soins peut paraître futile d'un point de vue curatif, quand aucune guérison n'est envisageable, les gestes susceptibles d'être posés peuvent cependant s'avérer importants et pertinents d'un autre point de vue, quand, par exemple, ils permettent au mourant et à ses proches de préparer et d'assumer leur deuil. Et l'inverse peut aussi être observé : le prolongement médicalement souhaitable de la vie peut s'avérer contre-productif d'un point économique et organisationnel, notamment quand on se bouscule aux urgences alors que l'institution manque de ressources. S'annoncent chaque fois des décisions difficiles et douloureuses.

Quels critères vont prévaloir dans ces prises de décisions ? Certes, prendre au sérieux du futile déroge aux normativités institutionnelles qui ont pour mission de guérir. Mais souvent le dérisoire, par sa résistance même, est un passeur privilégié des questions de sens qui échappent aux protocoles rationnels (Halliday 1997 ; Javeau 1998 ; Lemieux 2000). L'éthique qui questionne l'indéfini du sens devient alors primordiale.

La problématisation du mourir, trop rapidement esquissée ici, soulève de plus en plus de questions inédites. Qu'est-ce que survivre ? N'y aurait-il pas dans l'incertitude que soulève cette question un facteur incontournable de l'intensité émotionnelle vécue à l'approche de la mort ? Mourants, soignants, accompagnateurs voire simples témoins qui par écrans interposés sont mis en présence de la mort des autres, sont alors confrontés au mystère de leur propre vie. Et, quelles que soient les horreurs que leur parcours jusque-là leur ait fait connaître, ils choisiront vraisemblablement la vie tant qu'ils le pourront, ne serait-ce que pour l'imprédictible beauté de son mystère. L'ultime fragilité, que révèle l'expérience de la mort, appelle une sublime espérance. 


\section{Espérer}

"L'espérance, c'est ce qui me fait sortir de la mort. Encore faut-il l'avoir éprouvée, la mort " (propos de Denis Vasse dans Farin 2012, 28 min 40 s). La phénoménologie de l'acte de mourir nous met en présence d'une volonté de contrôle certes diverse dans ses expressions et ses moyens, selon l'état des sociétés et les types de collectivités humaines, mais visant toujours à lui donner du sens. Les manifestations d'espérance y sont fréquentes, mais plutôt que d'en former la trame principale, elles y apparaissent comme en filigrane, sortes d' « échappées belles », en quelque sorte, qui traversent l'ordre auquel on tente de soumettre l'humain, dans son mourir comme dans sa vie ordinaire. Ces « traverses » demeurent hétérogènes à la logique rationnelle-technique et parfois la perturbent. Elles relèvent d'une logique différente, comme le filet d'eau du ressac de la mer à travers les rochers, contrepartie de la vague écumeuse qui a balayé le littoral. Elles s'insinuent dans les dédales de l'ordre établi (Certeau 1980, 57). C'est en cela même qu'elles sont indicatrices et porteuses d'espérance : celle-ci ne consiste-t-elle pas à miser sur l'Autre, malgré tout, c'est-à-dire alors même que sont épuisés la rationalité, la force, l'ordre social, les montages idéaux de la culture ? L'espérance est une mobilisation de l'être : non pas un état stable mais une tension.

Mourir entraîne l'humain dans l'insondable gouffre de sa fragilité. Et l'espérance germe dans les profondeurs de ce gouffre. La réalité en est certes différente pour chacun mais elle concerne tous, individus et collectivités. Elle invite chacun à convoquer une instance autre, plus grande que soi. Des profondeurs, je crie vers toi, clamait ainsi Jonas, forclos dans les entrailles d'un monstre marin. Il rétablissait alors le lien salvifique, la tension entre son état et le mystère de l'Autre. Peut-on imaginer forclusion plus radicale que la sienne ? Banni de la ville, rejeté d'un navire, la mer ellemême ne veut pas de lui : il trouve son refuge dans l'intimité d'un monstre. Et peut-on trouver espérance plus sublime ? Son cri - même pas une parole - transcende l'espace et le temps.

Sublime espérance, dont le mouvement traverse les enclos des cultures. Elle est, certes, de l'ordre de l'utopie plutôt que de l'idéologie. Cette dernière, en effet, suppose un imaginaire commun à un groupe ou, dans la version marxiste canonique, une classe sociale, bref des traits culturels, des idéaux, tous objets reconnaissables et susceptibles d'être valorisés et mis au 
service d'une cause. L'utopie, par ailleurs, reste toujours à poursuivre : jamais atteinte, elle introduit à l'insaisissable. Elle est "enfouie dans nos âmes ", disait Ernst Bloch (1977, 305). Néologisme du XVIe siècle qui s'est imposé à partir de l'œuvre de Thomas More, mot latin dérivé du grec (topos: lieu), utopia peut aussi bien désigner un lieu de nulle part qu'un lieu de félicité, un non-lieu - ou-topos - ou un bon lieu - eu-topos éminemment désirable. Voilà bien que nous rencontrons encore une fois cette réalité propre à l'humain : «l'indéfini du sens». Cet indéfini s'inscrit au niveau le plus élémentaire de l'ordre symbolique, celui de la langue. $\mathrm{Ne}$ peut-on penser que l'autre monde, objet fuyant de l'espérance, est à la fois non-lieu et lieu de bonheur, lieu de nulle part et lieu du désirable ? Espacetemps qui se trouve, du même coup, intemporel: enraciné dans la concrétude des déterminations et de la finitude, il donne la possibilité d'aspirer à autre chose.

Sans quitter l'ordre des phénomènes, qui s'impose dès qu'on porte attention à quelque réalité, on peut alors suggérer des pistes pour une théologie de l'espérance. Celle-ci, en effet, ne conteste pas les conjectures anthropologiques. Elle n'est pas non plus un ajout qu'on pourrait greffer à la connaissance de l'humain à partir d'une science des Écritures ou d'une Révélation : elle en assume au contraire les éclairages et les nébulosités, les possibilités et les limites, dans un travail d'intelligence ${ }^{7}$ (nous en évoquerons plus loin quelques conditions) qui est lui-même un acte d'espérance.

La tension vitale à laquelle tout sujet est soumis est assez facile à discerner : elle est commune à tout vivant. Elle concerne le rapport du défini à l'indéfini qui se vit à tous les niveaux et en tous lieux. La fleur qui pousse au bord de la route est enracinée dans un terreau bien concret, riche ou pauvre, mais elle tend vers la lumière : sa beauté fugace manifeste cette tension, pour qui veut la contempler. Le nouveau-né tend vers le sein maternel : pour le reste de ses jours il cherchera l'altérité sans laquelle il ne saurait vivre. La coupure étant condition de sa venue au monde, l'humain comme

7 Entendons par ce terme le travail d'un sujet qui cherche à s'approprier les conditions de son existence. Ce travail, selon les conditions de la culture dans laquelle il opère, consiste à construire une représentation des phénomènes de façon à saisir au mieux leur structure et leur fonctionnement, dans la relativité de leur histoire et de leur devenir incertain. Ainsi considérée, l'intelligence n'est pas un état ou une chose, voire un don qu'on posséderait ou non, mais l'acte d'un sujet en processus qui doit réinventer constamment son rapport au monde, pour survivre. 
les autres animaux est mis en demeure, pour survivre, de reconstituer sans cesse du lien social. C'est là d'ailleurs l'enjeu de ses activités sexuelles - le mot dérive de secare, couper - quoi qu'il s'en avoue, même dans la pornographie qui marchande des morceaux de corps en guise de succédanés relationnels (Dufour 2009, 27). Et l'enjeu de cette quête, en fin de course, est rien de moins que l'identité, c'est-à-dire la possibilité d'être soi-même, parmi d'autres, là où chacun : «définit un emplacement singulier par l'extériorité de son voisinage » (Foucault 1969, 27).

Dès qu'on prend conscience de cette dynamique vitale, on rencontre le délicat et obsédant défi d'en rendre compte. Pour cela, chacun est mis en demeure de décliner son identité de façon à la rendre visible et saisissable par tous : elle signale, en effet, une place singulière mais exposée aux autres, à la manière d'un parterre de fleurs enclos pour sa protection et sa mise en valeur.

Cette dynamique identitaire fait donc du sujet espérant un être enraciné mais sans cesse en tension vers l'altérité. Elle est vérifiable tant chez les individus que dans les collectivités géographiques, politiques, communautaires ou associatives, quels qu'en soient les contenus et les formes. Hérités ou acquis au fil de son histoire, les traits identitaires représentent pour tout groupe, et a fortiori pour l'individu, le lieu propre, à la fois singulier et relationnel, de son être au monde. Chaque fois s'y joue l'enjeu du défini et de l'indéfini.

Le champ du défini, l'enclos, dès lors prend sens grâce au champ de l'Autre, un plus grand que lui, dans l'ouverture à l'indéfini du monde. On comprendra que cette tension constitutive de l'humain se trouve aussi dans l'Église et chacune de ces communautés qui se qualifient elles-mêmes, ou qu'on qualifie, de chrétiennes. Le réel de l'Église, avance ainsi Gérard Siegwalt (2016), ne se trouve ni dans ses institutions ni dans ses utopies, mais dans la tension dont elle vit: tension entre ses implantations, d'une part, et ses idéaux, d'autre part. D’un côté, plurielles et variées dans leurs formes, ses implantations témoignent de réalités éclatées tant sur le plan confessionnel que géographique et démographique. Parfois fertiles, parfois arides, ces terreaux laissent place à l'ivraie comme au bon grain. Par ailleurs, l'Église vit aussi de son idéal de pureté et l'utopie de la cité céleste. 
Elle est, littéralement, poussée désirante cherchant à témoigner du fait qu'» un autre monde est possible ${ }^{8}$.

Si l'Église est "proposition de vie », elle ne peut être réduite à l'un ou l'autre de ces pôles. Elle est quête de pureté mais le péché fait partie de sa vie et elle doit l'assumer. Cette capacité d'assumer est même un signe de sa santé. Mais l'utopie lui est aussi inhérente et doit être proclamée, même quand la distance entre l'idéal et la réalité semble abyssale.

Or cette distance, peut-être pas plus importante qu'en d'autres époques, est aujourd'hui devenue patente et elle est claironnée inlassablement par les médias, au service certes de bien d'autres intérêts. Mais du même coup, cela protège les croyants, théologiens et clercs en tête de liste, de la tentation du pouvoir à laquelle ils ont souvent sacrifié dans l'histoire. Aujourd'hui le théologien vit dans la misère (Certeau 1973), comparativement au médecin, au savant, au technicien dont les savoirs sont célébrés en vertu de leur reconnaissance sociale à l'intérieur des enclos culturels. Il ne dispose ni d'un $\mathrm{PPBS}^{9}$ à la manière des commis de l'État, ni de data-based evidence pour qualifier l'excellence de ses pratiques. Il est tributaire de ce que Michel de Certeau (1987) a appelé la faiblesse de croire. Qu'est-ce donc que l'espérance chrétienne ? La logique en est simple : alors que l'altérité est pour le monde menace de mort, le croyant, lui, «d'un geste déraisonnable, en attend la vie» (Certeau 1987, 262). En christianisme tout au moins, le rapport du croyant à l'espérance relève ainsi d'une véritable conversion épistémologique (Thibault 2004), geste déraisonnable, mais sensé.

L'expérience mystique représente un lieu majeur d'exploration de cet enjeu. C'est pourquoi elle cherche sans cesse des mots nouveaux pour rendre compte de son caractère fabuleux. Elle épouse en cela d'une façon particulièrement congruente les dynamiques de la modernité, notamment quand celles-ci donnent priorité à l'énonciation plutôt qu'à l'énoncé, ou encore quand elles incitent à conjuguer le geste esthétique et l'engagement éthique. Bref, elle transforme les régimes conventionnels du rapport au monde en terreaux pour des sujets en processus. Il est remarquable d'ail-

8 Slogan issu du forum social mondial de Porto Alegre (2001) et qui résume l'espérance du mouvement altermondialiste, aujourd'hui dans de multiples contextes. Voir Susan George (2004).

9 Planning Programming Budgeting System. 
leurs que cette expérience se concrétise volontiers en poésie, en actes créateurs, voire en réformes des institutions religieuses conventionnelles, traversant les codes établis pour y insuffler de la vie nouvelle. Elle s'acharne à poser, c'est-à-dire croire, qu'il y a de l'autre, fut-il indéfini et échappant à toute emprise culturelle. Elle fonde en cela une espérance qui s'avère, véritablement, poḯtique.

Est mystique celui ou celle qui ne peut s'arrêter de marcher et qui, avec la certitude de ce qui lui manque, sait de chaque lieu et de chaque objet que ce n'est pas ça, qu'on ne peut résider ici ni se contenter de cela. Le désir crée un excès. Il excède, passe et perd les lieux. Il fait aller plus loin, ailleurs. Il n'habite nulle part. Il est habité [...] par un noble je ne sais quoi ni ceci, ni cela, qui nous conduit, nous introduit et nous absorbe en notre Origine. (Certeau 1982, 411)

Certes, on pourra reconnaître là un phénoménal travail de sublimation. Or sublimer, justement, n'est pas un acte anodin mais bien une mobilisation vitale de l'être qui vise un supplément d'être. Cela pose, évidemment, la question de la "vérité ", une vérité qu'aucune obsession pour l'exactitude ne peut satisfaire puisqu'elle ne consiste pas à se rendre conforme à quoi que ce soit mais engage à assumer dignement l'angoisse de vivre, l'expérience des limites et la persévérance dans l'être. Il s'agit alors de prendre, avec d'autres, le risque de l'indéfini du sens. Ce courage d'être, comme l'a appelé Paul Tillich (1999 ; Lemay 2003), est récurrent chez les mystiques. Il a permis à un François d'Assise, pour s'en tenir ici à ce seul exemple, de déchirer ses vêtements bourgeois pour développer une «liberté intérieure purifiée de ses investissements fusionnels» (Charron 1992). Il demande de choisir de vivre plutôt que macérer et gémir. Quand le caractère opportun de tels gestes devient évident dans le monde contemporain, à l'encontre des énonciations robotisées de " vérités » aplanies à ras de données probantes, il fait possiblement valoir "un autre esprit de vérité », un «courage de la vérité »(Sadin 2018, 31, citant Foucault 2016) qui se compromet dans l'indéfini du sens.

Ce courage, faut-il insister, ne consiste pas à nier le corps : pulsion oblige. Tout en tenant compte de ses enracinements, il remet sans cesse le sujet en mouvement, réinvente sa condition de sujet en tension pour l'ouvrir à « une jouissance jusque-là inaperçue » (Cliche 2016, 25) parce qu'elle ne doit rien, justement, aux calculs et fonctions utilitaires nécessaires à l'ordre des choses. C'est cette jouissance que la sculptrice américaine Kiki 
Smith a illustrée dans ses représentations de Marie-Madeleine : entravée, enchaînée au sol, elle est toute tendue vers l'Autre ${ }^{10}$, dans une nudité qui n'a rien de la marchandise érotique mais représente, à la manière de la statuaire grecque, la dignité du héros n'ayant pas besoin d'autre parure que la vérité de son corps sublimé. Dès lors la posture névrotique reconnaissable dans la sublimation, loin d'être réductible aux nosographies psychiatriques, est elle aussi une manifestation de l'espérance : typiquement humaine, elle permet au sujet de négocier ses limites pour continuer d'exister.

Certes, cela produit aussi de la souffrance, notamment quand l'idéal mobilisateur s'avère hors de portée, par exemple quand des intervenants en situation d'urgence sont confrontés à des scènes insoutenables, à l'aveuglement et l'absurdité de la violence. L'obsession d'être soi en performant toujours mieux et davantage peut ainsi générer une souffrance chronique quand le travailleur ordinaire "fait l'exploit des anciens gladiateurs, mais sans la gloire », comme le note Alain Ehrenberg (1991, 173 ; 1998). Pourtant ce travailleur, comme le migrant, reste un être d'espérance. Il cherche à améliorer son sort et celui de ses proches.

Que se passe-t-il, en réalité, sinon que l'accès à l'ouverture indéfinie du sens lui est refusé et que ses efforts de survie, sans cesse repris, sont chaque fois ramenés à la finitude de son environnement culturel ? On touche là sans doute le nœud de la question : espérer est un verbe performatif. Dire «j'espère » est en soi un travail d'ouverture sur l'altérité qui met l'être en mouvement. Comme la foi sa grande sœur, l'espérance est réélection d'un Autre que les grandes traditions monothéistes ont d'ailleurs voulu sans visage - allant jusqu'à en détruire et interdire les images - et au nom imprononçable, comme en témoigne le tétragramme.

Cet Autre est irréductible aux formations de l'imaginaire et du symbolique. Or, pour nourrir le désir de dépassement du sujet, il faut l'appréhender, le faire advenir au langage : on ne peut alors se passer de lui donner noms et images, de le représenter de mille et une façons. Et dès lors, on l'intègre à l'ordre de la culture, on l'incorpore dans le croyable disponible et les enclos de la culture. Situation aporétique ? Sans doute, mais nécessaire, les humains n'étant pas des anges. Aussi, le sens commun manque-t-

10 On trouvera plusieurs versions de «Mary Magdalene» sur le site :

https://commons.wikimedia.org/wiki/Category:Mary_Magdalene_by_Kiki_Smith_in_ L\%C3\%BCbeck\#/media/File:Maria_Magdalena_Burgtorkloster.jpg. 
il rarement de ramener aux «vraies affaires " - le "terrain des vaches", disaient les ancêtres paysans des gestionnaires contemporains - ceux et celles qui se risquent quelque peu sur les territoires de l'Autre. Du même coup, plus on s'enferme dans les enclos de la culture, plus s'exaspère le mal de vivre.

C'est pourquoi l'espérance s'avère une véritable éthique de la marche. Il s'agit, à chaque pas, tout en s'inquiétant des conditions du sol, de se rendre sensible à la beauté du paysage. Là où le geste esthétique et le geste éthique coïncident, le sujet qui en est l'agent ne peut que refuser l'autorité $\mathrm{du}$ fait et lui opposer une posture "atopique, révolutionnaire, "poiétique” » (Certeau 1982, 269). Est beau, dès lors, ce que rien n'autorise.

Nous retrouvons ici non seulement la Madeleine et les Anges inconscients ci-haut évoqués, mais une multitude d'œuvres d'art contemporaines ou anciennes qui travaillent à ré-élire ${ }^{11}$ la vie, en quelque sorte, dans l'expérience de la mort. En témoignent, notamment en musique, non seulement les Requiem de Mozart, Berlioz, Verdi, Fauré, Duruflé, pour ne citer que les plus classiques, mais plus près de nous ceux de Roman Maciejewski (1960), Krzysztof Penderecki (1984), Andrew Lloyd Webber (1984), Karl Jenkins $(2005)^{12}$ - qui présentent des œuvres non seulement monumentales mais porteuses, par leur seul langage musical, d'un retour réflexif sur la condition humaine. Et que dire de la peinture, de la littérature, du cinéma... ?

Travail d'espérance, le deuil ne consiste pas à remplacer une relation perdue par une autre, mais à redistribuer les enjeux du désir en tenant compte du manque inscrit dans la communauté humaine. Il concerne donc, en premier chef, le sujet en devenir. Survivre suppose ainsi de réapprendre l'intersubjectivité qui fait de chacun un être de relations. C'est bien un mouvement de l'être qui assume ses limites, un mouvement pour sortir de l'aliénation qu'imposerait, autrement, l'enfermement dans le même, l'enfer des convoitises où chacun s'exaspère en déchirements internes (Chamberland 1986, 20). L'être humain, dans le langage, bute sur le

11 Ce qui est aussi une étymologie possible du mot religion : à côté de religare (relier) et relegere (relire), re-eligere, ré-élire l'Autre sur lequel on porte le regard constitutif du sens. On la trouvera notamment chez Thomas d'Aquin, dans son traité sur la vertu de religion.

12 Liste non exhaustive. Une revue par BJ's Requiem Database en 1999, recensait 284 requiem issus de la créativité de 265 compositeurs, approximativement $80 \%$ de ceux-ci étant du vingtième siècle. 
défaut du signifiant : les signes sans cesse laissent à désirer. Il faut donc transcender l'ordre des choses pour continuer de vivre. Enjeu, sublime, de l'espérance.

\section{Vivre}

L'espérance invite à traverser les limites, celles des clôtures psychiques et morales qui conditionnent la marche des sujets comme celles des enclos culturels qui proposent buts et objectifs à réaliser. Elle est un pousse-à-vivre dans la montée du désir.

Mais qu'est-ce que vivre ? Qu'est-ce qui, dans le fait de vivre, rend l'espérance nécessaire ?

L'Autre échappant aux définitions qui pourraient le mettre en captivité, les mobilisations du sujet sont sans terme. Elles renvoient ainsi à la conception spinozienne du désir : " persévérer dans l'être », un être qui est ouverture à l'indéfini du monde, du cosmos tel que connu, ouverture à l'altérité que son dépassement laisse deviner. C'est d'ailleurs la raison pour laquelle le désir apporte la béatitude, une béatitude qui «n'est pas la récompense de la vertu mais la vertu même » (Spinoza 1999, proposition 42) au point que c'est parce que nous éprouvons de la joie, dans la montée du désir, que nous pouvons contrôler quelque peu nos pulsions mortiferes. L'expérience de la mort mettant en cause l'irréductibilité de ce désir, elle impose à l'être de reconnaître les fondements de son désir, voire son caractère connaturel, conatus, disait encore Spinoza.

La prière, chez les croyants et souvent aussi chez les non-croyants, présente un espace-temps particulièrement prégnant de cette réalité du désir. Prier, en effet - la dynamique en est particulièrement vérifiable dans la prière de demande — c'est s'autoriser à laisser monter son désir, dans un mouvement qui le met à l'épreuve de l'altérité et en teste la vérité.

Il y est donc question de vérité. Or on sait que celle-ci, pour l'humain tributaire du langage, n'est jamais évidente et toujours à chercher. Elle est sans cesse balafrée par les défauts du signifiant. Jacques Lacan aimait dire quelle est "pas toute", autrement dit sans cesse objet de quête (Causse 2018, voir en particulier le chapitre 5 : «Qu'est-ce que la vérité ?»), à l'encontre des ostentations et narcissismes qui prétendent la posséder et sont, en réalité, des pousse-à-la-mort. Quels que soient son intelligence et son capital culturel, l'humain vit dans le mi-dire : cela marque l'ensemble 
de ses rapports au monde, tant dans son agir que dans ses discours. N'estce pas saint Paul qui avoue : «Je ne fais pas le bien que je veux, mais je fais le mal que je ne veux pas » (Romains 7, 19). Dans le commentaire qu'il donne de Lacan, Alain Badiou précise que la vérité dont il est question se caractérise foncièrement par son impuissance, si bien que l'amour de la vérité doit être "amour de cette impuissance, de cette faiblesse " (Badiou 1992, 197 ; Causse 2015). Autrement dit, c'est un amour qui aime la vérité en tant qu'elle est manquante ou, en termes freudiens, une vérité inscrite dans la castration.

En tenir compte permet de mieux apprécier l'importance de la prière qui, dans l'antre même de la mort, fait entendre une voix s'adressant à un autre et en attend une réponse, faisant ainsi advenir dans le langage un face à face : "Du ventre de la mort, j'appelle au secours : tu entends ma voix. [...] Si bien que je me suis dit : je suis chassé de devant tes yeux [...] Et ma prière parvient jusqu'à toi, jusqu'à ton temple saint » (Jon 2, 3.5.8). Dès lors inventés et sans cesse réinventés pour s'inscrire dans l'ordre symbolique, le visage et la voix de l'Autre témoignent d'un échange, c'est-à-dire d'une parole reçue et adressée, d'une socialité particulière. Cet échange autorise et façonne, bien sûr, l'identité du sujet désirant.

Le désir s'inscrit ainsi dans un jeu de reconnaissances mutuelles. Cela ne va pas sans paradoxes ni sans dangers. Le sujet de la parole s'avérant mouvement, mobilisation désirante, montée vers l'Autre, ni lui ni cet Autre ne peuvent être fixés dans une image, tant et si bien que l'arrêt sur image, comme au cinéma, risque de brûler la pellicule : «Hors parole, là où il n'y a pas de sujet envisagé, ni même envisageable, du même coup, n'importe quelle forme animale, minérale ou mythique peut être substituée au visage manquant» (Vasse 1983, 136). Quand Narcisse se prétend parvenu à la fin de l'histoire, c'est qu'il travaille inconsciemment à programmer la fin de l'humanité.

À l'opposé d'une telle autosatisfaction pathologique, la prière introduit au nœud gordien de l'art de vivre : elle manifeste la réalité d'un sujet, être parlant qui s'adresse à un autre dont le visage lui échappe. Mais pour établir cet autre dans un échange effectif, elle a besoin de lui donner visage, de le (re)présenter. Ce n'est pas là une opération innocente : donner visage à l'altérité, c'est l'inscrire dans la culture, l'amener à participer aux jeux de société dont celle-ci se repaît, bref c'est la réduire pour lui donner valeur relationnelle ici et maintenant, bref identité relative. 
Le moins qu'on puisse dire, c'est que le défaut du signifiant provoque alors du malentendu, dans tous les sens du terme : distorsion du message selon les conditions de sa transmission et de son écoute, distorsion du désir par son aplatissement au terre-à-terre des enjeux culturels. C'est pourquoi prier, comme l'écrit encore Denis Vasse (1971, 282), «c'est tenter d'entendre la Parole dans laquelle nous vivons et que la nôtre cherche à couvrir du bruit de ses mots... C'est arrêter de parler pour écouter quelqu'un qui veut faire entendre sa parole ». Et dès lors, une altérité peut se dévoiler, une parole originelle en ce qu'elle devient fondatrice de l'humain dans son existence désirante. Quand cela advient, il y a renversement, conversion épistémologique, "premier pas de la libération de notre corps et de notre parole» (Vasse 1971, 285).

L'Autre ainsi inscrit dans le langage doit certes être l'objet d'une grande prudence et de soins constants, car la propension humaine est de sans cesse le ramener à ras le sol, là où il peut être opérationnalisé, parfois au service d'une cause noble, par exemple un idéal national ${ }^{13}$, souvent en fonction des commerces et jeux de pouvoir qui animent les enclos culturels. Par contre, il peut se faire connaître en toutes choses, dans le sentiment océanique comme dans le fatras de la ville, dans le berceau du nouveau-né comme dans le corps supplicié de l'adulte, dans les visages adolescents comme dans ceux des itinérants, migrants, éclopés, malades, vieillards...

Prier est « un mouvement de l'âme dans un moment de vérité » (Rancé 2012, 69) : un pousse-à-la-vérité, pourrions-nous dire, antidote des pousse-à-la-mort de la séduction. "Mouvement de l'âme ", c'est-à-dire animation par laquelle l'être en devenir cherche son plein épanouissement, audelà de tous les modèles de « réalisation de soi » que peuvent lui proposer les idéaux du moi. «Moment de vérité » : insatiable, parce que cette vérité reste toujours à chercher. Cela implique pour le sujet de se ressaisir, de se débarrasser des encombrements mondains et de repositionner son rapport au monde, c'est-à-dire à l'altérité dans toutes ses occurrences : l'Autre incommensurable qui l'appelle et à qui il fait confiance, l'autre en lui-même

13 Pour un exemple contemporain et rigoureusement séculier, voir l'hommage aux deux fusiliers marins tués lors d'une opération de libération d'otages au Burkina Faso, le 10 mai 2019. Le Président de la France y répète, dans un discours magistral, la noblesse d'une « cause plus grande que vous, celle de la France, celle de la liberté ». En ligne https://www.lejdd.fr/Politique/hommage-aux-deux-soldats-francais-tues-voici-le-discours-demmanuel-macron-en-integralite-3898539 (consulté le 20 avril 2020). 
qui travaille le plus intime de son être (le for intérieur, disait Maître Eckhart, où « Dieu lui-même n'a encore jamais pénétré de son regard » [1974, 56]), et les autres, le prochain plus ou moins lointain.

Sur cette piste, on en arrive rapidement au mystère, ce mystère dont Luther disait que "dans la mesure où Dieu se cache et ne veut pas être connu de nous, il ne nous concerne pas. [...] Mais dans la mesure où il s'est incarné et révélé à nous par sa Parole, nous avons à nous occuper de lui » (Luther 1958, 110). En conséquence, il convient encore de travailler à " débarrasser Dieu de Dieu »(Causse 2018, 53), parce que si, d'une part le réel qu'en pointe le concept invite à une quête illimitée, l'imaginaire, lui, le fige immanquablement. Dès lors, concept parmi d'autres concepts, l'Autre devient enjeu des luttes fratricides pour le contrôle des enclos culturels.

"Y a-t-il un risque à nommer Dieu ?", demande ainsi André Gounelle (2004, 29). Parler de Dieu ne revient-il pas à « le mondaniser, c'est-àdire nier sa transcendance et la distance qui nous en sépare » ? Or c'est dans ce risque de la parole, actualisation du désir, que prend consistance l'acte de vivre : risquer un rapport à l'altérité, à l'ineffable, rapport que l'on sait insatisfaisant, qu'il faudra donc reprendre inlassablement, mais qui permet d'effectuer un pas de plus, un paso mas alla, bref de rester mobilisé.

Vivre, espérer, c'est ainsi tenter sans cesse de quitter l'enfer des choses et en oublier au moins pour un temps les déchirements internes, se dérober autant que possible des enfermements qui réduisent le désir à de l'» agitation célibataire dans le cercle étroit des semblables " (Chamberland 1986, 14 et 20), bref, récuser le «narcissisme grégaire »(Dufour 2007) qui incite chacun à se penser libre alors qu'il est télécommandé par les canons de son environnement, voire, dans le monde sous l'égide néolibérale, par « une puissante et invisible main de fer» (Dufour 2007, 19). Non seulement épistémologique, la conversion s'avère alors éminemment pratique : chercher Dieu... en toutes choses... malgré tout.

Les mises en scènes du symbolique, «vie des signes dans la vie sociale» (Saussure 1975, 33), concernent chacun dans son être au monde, c'est-à-dire sa réalité de sujet enraciné, enchaîné à une histoire singulière mais en processus d'ouverture, incarné et par là assujetti aux violences du monde mais en quête de libération. Tant qu'il reste pris dans les convulsions des enclos culturels, le sujet est incité, voire forcé à renoncer à son être propre pour réaliser les idéaux du moi auxquels il est assigné. C'est 
l'enfer. Mais c'est là, au creux de ses conditions vitales, que devient essentielle l'imprédictible beauté du monde et de ses mystères. Aux impératifs de l'utilitaire - l'uti, pour emprunter le vocabulaire augustinien — il faut, non pas opposer mais conjuguer le frui. «Frui : jouir au sens de la réjouissance, de la joie, [de] ce qui ne sert à rien, qui est là pour rien, sans raison aucune, sans signification »(Causse 2018, 99). Et ajoutons-nous, au sens de s'autoriser à contempler, acte qui ne sert effectivement à rien dans les enfermements culturels obsédés de production-consommation, mais s'avère hors de prix dans les quêtes de sens.

Pourquoi la beauté ? Parce que sans elle, comme le note encore Certeau (1982, 269), il n'y a plus que des corps, c'est-à-dire de la bestialité. Si, complètement pris dans la mondialisation, on ne peut plus voir la beauté du monde qu'expose par ailleurs sa mondialité, c'est-à-dire la différence inscrite dans la multiplicité des visages donnés à contempler, on s'engage dans un cul-de-sac totalitaire où la prédation, la violence et la destruction déterminent le sort de l'humain.

Sur quoi se fondent les élans migratoires ? Bien sûr : la guerre, la terreur, la peur, la souffrance économique, les désordres du climat... Mais aussi : sur l'appel secret de ce qui existe autrement. La plupart des migrants ont identifié le lieu d'une arrivée, qu'ils ont choisi ou qu'a choisi pour eux leur perception du monde. Ils sont habités par une vision surgie de la mondialité. Sans doute subjective partielle partiale, aliénée par les forces dominantes qui nous formatent l'imaginaire, mais vision tout de même. En eux, elle a rompu les verticalités du paysage, élargi au-dessus des frontières leur territoire vital. L'a installé dans l'ardeur d'une promesse. C'est cette vision qui rend leur élan impérial, tendu entre la vie et la mort et s'acceptant ainsi. (Chamoiseau 2018, 67)

\section{Cette vision autorise à vivre.}

En nous ouvrant à la mondialité, en aidant l'imaginaire relationnel à faire intelligence en nous, le chaos peut devenir fécond. L'imaginaire relationnel (porté aux bienveillances) y trouve l'occasion d'un humanisme bien plus large, plus profond et plus humble, mieux conforme au vivant... La dynamique qui va de soi à l'Autre, de l'Autre vers soi, en sort privilégiée, et cette notion de l'Autre se voit ouverte au-delà des seules présences humaines. (Chamoiseau 2018, 71)

Devant les enjeux de la beauté concrétisée ici par l'espérance des migrants, le premier défi, incontournable, est celui de prendre soin, de façon à 
procurer la meilleure assise possible à l'espace nécessaire à l'advenue et à la progression du sujet désirant. Cet espace, qui structurellement fait brèche dans la culture, est intersubjectif. Lieu de rencontre du mystère d'un sujet avec le mystère d'un autre sujet, il promeut des échappées dans la tension constitutive de l'humain, échappées qui sont comme autant d'éclats lumineux sur les vagues, dans la nuit. Cela se trouve communément non seulement dans l'accompagnement spirituel de fin de vie où nous avons pu l'observer à l'occasion (Lemieux 2018) mais aussi, certes moins communément, en éducation (Paturet 2007, 62) quand le maitre humanise le savoir, montre son caractère perfectible, exposé au doute et ouvert au désir de partager quelque chose de plus grand que soi, au-delà des objectifs institutionnels d'acquisitions (et de consommations) socioculturelles.

Cet espace de vie suppose un lieu réservé, intime en quelque sorte, où peut se déployer la responsabilité du sujet, espace moins structuré par des programmes et protocoles d'entrainement que par l'appel de l'altérité. C'est dire qu'il sollicite à la fois la liberté et la vérité du désir, deux instances aux limites elles-mêmes indéfinies, et qu'il donne lieu à des enjeux éthiques et épistémologiques fondamentaux, tant au plan théologique que strictement anthropologique.

Ce prendre soin doit évidemment s'appliquer à tous les stades de la tension dans laquelle se concrétise l'espérance, et en particulier ses pôles : l'enracinement du sujet, l'envol vers l'altérité.

Prendre soin de ses enracinements, d'abord, parce que l'identité suppose un emplacement singulier, condition de possibilité du développement des relations avec les autres, socle à partir duquel les mobilisations peuvent advenir. Toute identité humaine, avec son histoire, sa langue maternelle, sa vision du monde, ses croyances, etc., est un compendium unique d'expériences. Briser les identités, comme l'ont pratiqué les barbaries historiques notamment dans le colonialisme moderne, est pour cela un véritable crime contre l'humanité : c'est nier le caractère spécifiquement humain de l'être, sa capacité de mobilisation à partir de sa singularité.

Mais s'il faut se préoccuper des conditions physiques et culturelles de l'être au monde, il faut aussi prendre soin de l'Autre, pour éviter que son image soit balafrée par les intérêts circonstanciels des enclos, qu'elle se fixe en idoles de pierre ou de chair, bref que le désir de vivre soit perverti 
dans les objets donnés à consommer. "Prendre soin de son âme ", enseignait Michel Foucault (2001), suivant la consigne de Socrate au jeune Alcibiade.

En fait, l'exploration des territoires de l'Autre exige, à tout niveau, un travail d'intelligence dans lequel la rigueur ne cède rien à la créativité. Insistons sur le fait qu'il s'agit bien d'un travail : elle aussi tributaire des défauts du signifiant, l'intelligence n'est pas un état mais est bien un labeur, puisque la vérité reste sans cesse à mettre en lumière.

Luis Sepúlveda (1992), survivant des années sombres du Chili et réfugié dans la jungle amazonienne, en explore les conditions dans son roman Le vieux qui lisait des romans d'amour. On peut en dégager trois niveaux d'exigences, qui ne garantissent rien, mais rendent possible la survie dans les territoires indéfinis de l'altérité. Le premier renvoie à la connaissance empirique du milieu à explorer, au mieux que cela est possible (ce qu'on appelle la phénoménologie dans les pratiques scientifiques). Dans la jungle il s'agit de la flore (qui peut aussi bien nourrir qu'empoisonner), de la condition des sols, de la pluviosité et de l'ensoleillement, et bien sûr de tout ce qui grouille sous la canopée : serpents et jaguars, faune furtive ou agressive, sans compter les autres humains, chasseurs en quête de trophées, bandits en cavale, explorateurs, braconniers, orpailleurs, missionnaires, anthropologues... ni les aborigènes avec leur culture et leur expérience propre du milieu.

Mais ce premier niveau est insuffisant. Il faut aussi l'empathie, ce qui manque aux intrus motivés par la quête du profit. Chasseurs, chercheurs d'or et prospecteurs peuvent très bien connaître la jungle mais ils en deviennent victimes dès lors que leur pulsion prédatrice prend le dessus sur le respect de son altérité. Il leur manque la capacité de se mettre à la place de l'autre, d'appréhender l'environnement en passant par son regard et, indépendamment de toute attirance ou répulsion (les deux pouvant s'avérer mortiferes), de chercher à saisir la logique qui préside à sa quête de survie. Fut-il sauvage, l'autre est un être vivant qui tend, lui aussi, à pérenniser ses conditions d'existence.

Enfin, troisième condition de l'intelligence : l'esprit critique. Encore ici, une conversion épistémologique s'impose : il ne s'agit pas de critiquer l'autre - à quoi cela pourrait-il servir dans la jungle ? - mais de prendre conscience des possibilités et limites de ses propres vues et aveuglements, 
bref du caractère relatif de toute posture tenue devant le mystère de l'altérité.

C'est là aussi un travail sans cesse à recommencer qui renvoie à Grégoire de Nysse cité en introduction. La raison en est simple. Elle a été lapidairement et crument prise en compte par Marx, en pleine modernité, quand il a dénoncé le fait que la religion détachait les réalités humaines de leur base profane "pour aller se constituer dans les nuages en royaume autonome » (Marx 1972, 29). Or c'est précisément là que conduit l'exploration des territoires de l'altérité négligeant l'esprit critique : produire des royaumes autonomes, des fantasmes - ou systèmes de croyances - qui finissent par occuper complètement la conscience et occulter l'altérité, au profit d'» appartenances » intégrant l'individu à des collectivités supposément capables de lui garantir sens et puissance (Durkheim 1960, 327). De tout temps le montrent, souvent dramatiquement, les sectes dans lesquelles se coltinent les âmes errantes, eyes wide shut ${ }^{14}$.

Confrontée à l'indéfini du sens, l'intelligence est appelée à servir non pas le pouvoir mais la beauté, c'est-à-dire ce qui fait signe du dépassement de la mort.

En bref, l'ouverture à l'altérité, celle de la mondialité, des multiples visages de l'humain, celle de l'Autre sans visage, est un pare-feu contre la mort dont la pulsion habite non seulement chacun dans sa singularité mais aussi le sens commun cultivé dans les enclos culturels.

Et voici ce que provoque [le] planétaire assombrissement : l'exclusion, le rejet, la violence, la bêtise, la haine et l'indécence qui fermentent de partout, qui s'amplifient dans des boucles d'algorithmes et de réseaux sociaux, qui explosent dans la horde instinctive des médias que ces réseaux fascinent jusqu'à rendre mimétique. Cet effondrement engendre une perte de l'éthique, et quand l'éthique défaille, c'est la beauté qui tombe. (Chamoiseau 2018, 20)

14 Film de Stanley Kubrick (1999) tiré d'une nouvelle d'Arthur Schnitzer (2002). 
Quand l'éthique défaille, c'est la beauté qui tombe. Et quand la beauté tombe, c'est l'humain qui sombre. Tel est l'enjeu de la sublime espérance qui mise sur l'imprédictible beauté des battements tragiques des cœurs humains.

\section{Références}

ALLARD, M. (2001), Éthique et religion. Une lecture du traité de la religio dans la Summa theologiae de Thomas d'Aquin, Thèse de doctorat en théologie, Université Laval.

ARIES, P. (1967), «La mort inversée. Le changement des attitudes devant la mort dans les sociétés Occidentales ", Archives européennes de sociologie, 8, p. 169-195.

(1975), Essais sur l'histoire de la mort en Occident du Moyen Âge à nos jours, Paris, Seuil.

BAdiou, A. (1992), Conditions, Paris, Seuil.

Baudelaire, C. (1962), "Correspondances", dans C. Baudelaire, Les fleurs du mal, Paris, GF-Flammarion.

BEAUVOIR, S. de (1964), Une mort très douce, Paris, Gallimard.

BERGER, P. (1971) [anglais 1967], La religion dans la conscience moderne, Paris, Centurion.

Bloch, E. (1977) [allemand 1923], L'esprit de l'utopie / trad. par A.-M. LANG et C. PIRON-AUDARD, version de 1923 revue et modifiée, Paris, Gallimard.

Caenepeel, D. (2007), « Penser une éthique du soin préventif en psychiatrie », Thèse de doctorat en théologie, Université Laval.

CAusse, J.-D. (2015), «L'incomplétude de la vérité et la force du témoignage ", Laval théologique et philosophique, 71, numéro 1, p. 15-29. (2018), Lacan et le christianisme, Paris, Campagne-Première.

Certeau, M. de (1973), «La misère de la théologie, question théologique », La Lettre, 182, p. 253-265.

(1980), L'invention du quotidien. 1. Arts de faire, Paris, Union générale d'éditions. 
(1982), La fable mystique XVI'-XVII siècle, Paris, Gallimard.

(1987), La faiblesse de croire, Paris, Seuil.

Chamberland, P. (1986), «La fin des morales. Pourquoi le devenir nihiliste est-il inévitable ?", Les cahiers éthicologiques de l'UQAR, 13, p. 525.

Chamoiseau, P. (2018), Frères migrants, Paris, Seuil.

Charron, J.-M. (1992), De Narcisse à Jésus. La quête de l'identité chez François d'Assise, Montréal / Paris, Paulines / Cerf.

Cliche, A. E. (2016), Tu ne te feras pas d'image, Montréal, Le Quartanier.

Derrida, J. (2004), "Je suis en guerre contre moi-même», Le Monde, 19 août, p. 8-9.

Dufour, D.-R. (2007), Le Divin Marché. La révolution culturelle libérale, Paris, Denoël. (2009), La Cité perverse. Libéralisme et pornographie, Paris, Denoël.

Duneton, C. (2004), Le Monument, Paris, Balland.

DurkHeIM, E. (1960) [1912], Les formes élémentaires de la vie religieuse, Paris, Presses universitaires de France.

Ehrenberg, A. (1991), Le culte de la performance, Paris, Calmann-Lévy. (1998), La fatigue d'être soi. Dépression et société, Paris, Odile Jacob.

ELIzONDO, V. (2002), El caminar del Galileo. Promesa México-Americana, San Antonio / Texas, Mexican American Cultural Center.

FARIN, M. (2012), Sainte Thérèse de Lisieux. Histoire printanière d'une petite fleur blanche, film, Paris, Comité français de radio-télévision, $59 \mathrm{~min}$.

FouCAUlt, M. (1969), L'archéologie du savoir, Paris, Gallimard. (2001), L'herméneutique du sujet. Cours au Collège de France, 19821983, Paris, Gallimard / Seuil. (2009), Mystère et vérité, Paris, Gallimard / Seuil. 
FUCHS, E. (1998), « Problématique du salut à l'âge de la post-modernité », Revue d'éthique et de théologie morale "Le Supplément », 207, p. 139148.

Gounelle, A. (2004), Parler de Dieu, Paris, Van Dieren.

HALIDAY, R. (1997), « Medical Futility and the Social Context », Journal of Medical Ethics, 23, p. 148-153.

GeERTZ, C. (1986), Le sens commun en tant que système culturel. Savoir local, savoir global. Les lieux du savoir, Paris, Presses universitaires de France (Sociologie d'aujourd'hui).

GeORGE, S. (2004), Un autre monde est possible si..., Paris, Fayard.

GRÉGOIRE DE NYSSE (2009), La colombe et la ténèbre, textes extraits des homélies sur Le Cantique des cantiques, Paris, Cerf.

Javeau, C. (1998), Prendre le futile au sérieux. Microsociologie des rituels de la vie courante, Paris, Cerf.

KÜBlER-Ross, E. (1975), Death the Final Stage of Growth, Englewood Cliffs, New Jersey, Prentice-Hall.

Kubrick, S. (1999), Eyes Wide Shut, film, USA / UK, Warner Bros, 159 min.

LE MAY, J.-P. (2003), Se tenir debout. Le courage d'être dans l'œuvre de Paul Tillich, Québec / Paris, Presses de l'Université Laval / L'Harmattan.

LemieuX, R. (1999), "Sécularités religieuses. Syndromes de la vie ordinaire ", Cahiers de recherche sociologique, 33, p. 19-50. (2000), «L'art d'apprêter les restes. Lieux et enjeux éthiques de l'intervention auprès des humains ", Laval théologique et philosophique, 56, p. 509-529.

(2018), «Les soins spirituels dans les institutions de santé. Futilité, utilité, nécessité ? », Cahiers francophones de soins palliatifs, 18, p. 3041.

Luther, M. (1958), « Du serf arbitre », dans M. LuTHER, CEuvres, t. 5, Genève, Labor et Fides.

MAître ECKHART (1974), Sermons / trad. par J. AnCELET-Ustache, Paris, Seuil, 1974. 
MARX, K. (1972), "Thèses sur Feuerbach », dans K. MARX et F. EnGELS, L'idéologie allemande, Paris, Éditions sociales, p. 25-33.

Montaigne, M. de (1962) [1580], Essais, Paris, Gallimard (Bibliothèque de la Pléiade 14).

MORE, T. (1987) [1516], L'utopie ou Le Traité de la meilleure forme de gouvernement, Paris, Flammarion.

MOUNIER, E. (1962), Introduction aux existentialismes, Paris, Gallimard.

PATURET, J.-B. (2007), De la responsabilité en éducation, Toulouse, ERES, 2007.

PÉGuY, C. (1992), Le Porche du mystère de la deuxième vertu, Paris, Poésie / Gallimard, http://fr.wikisource.org/wiki/Le_Porche_du_myst\%C3\%A8re_de_1 a_deuxi\%C3\%A8me_vertu\#mw-head

POLANYI, K. (1983) [anglais 1957], La Grande transformation. Aux origines politiques et économiques de notre temps, Paris, Gallimard.

RANCÉ, C. (2012), Prenez-moi tout mais laissez-moi l'extase, Paris, Seuil.

SADIN, E. (2018), L'intelligence artificielle ou l'enjeu du siècle. Anatomie d'un antihumanisme radical, Paris, L'échappée.

SAUSSURE, F. de (1975), Cours de linguistique générale, Paris, Payot.

SChnitZer, A. (2002), La Nouvelle rêvée, Paris, Le Livre de Poche.

Sepúlveda, L. (1992), Le vieux qui lisait des romans d'amour, Paris, Métailié.

SiegWalt, G. (2016), Le défi ecclésial. Une voix protestante pour la réalisation de l'Église. Écrits théologiques IV, Paris, Cerf (Patrimoines).

SpINOZA, B. (1999), L'Éthique, Paris, Seuil (Points essais), http://classiques.uqac.ca/classiques/spinoza/ethique/ethique_de_Spinoza.pdf

SutTer, L. de (2016), Théorie du kamikaze, Paris, Presses universitaires de France.

TILliCH, P. (1999), Le courage d'être, Québec, Presses de l'Université Laval.

Thibault, D. (2004), La mystique chrétienne. Du désir d'unité au désir de l'Autre, une conversion épistémologique, Thèse de doctorat en théologie, Université Laval. 
Thomas, L.-V. (1975), Anthropologie de la mort, Paris, Payot.

Tronto, J. C. (2009), Un monde vulnérable. Pour une politique du care, Paris, La Découverte.

VASSE, D. (1971), « Prière et structure de l'homme », Carmel, 8, p. 276-295, http :/www.denis-vasse.com/wp-content/uploads/2009/09/1971Prière-et-structure-de-lhomme-D.VASSE-Carmel-VIII.pdf (1983), Le poids du réel, la souffrance, Paris, Seuil.

\section{Résumé}

L'espérance est cette vertu propre à l'humain qui lui permet de concevoir un monde différent et l'engage à travailler à son avènement. Que peut-il advenir, dès lors, dans ce passage ultime de sa vie où il est confronté à la mort? Trois facettes de la question sont explorées : comment se présente désormais l'expérience de la mort? Comment s'y greffe l'espérance, anthropologiquement et théologiquement ? Comment le fait de vivre appelle une ouverture à l'altérité qui est comme pare-feu à l'encontre des pulsions mortiferes? Le défi de la vie n'est-il pas dès lors d'espérer, malgré tout?

\section{Abstract}

"Ultimate fragility and sublime hope: the experience of death". Hope is the virtue of the human being that allows him to conceive a different world and commits him to work towards his advent. What can happen, then, in this ultimate passage of his life when he is confronted with death? Three facets of the question are explored: how does the experience of death now look? How is hope, anthropologically and theologically, added to it? How does living call for an openness to otherness that is like a firewall against mortal impulses? Isn't the challenge of life hope, in spite of everything? 